\title{
Entanglement beyond subsystems
}

\author{
L. Viola, H. Barnum, E. Knill, G. Ortiz, and R. Somma
}

\begin{abstract}
We present a notion of generalized entanglement which goes beyond the conventional definition based on quantum subsystems. This is accomplished by directly defining entanglement as a property of quantum states relative to a distinguished set of observables singled out by Physics. While recovering standard entanglement as a special case, our notion allows for substantially broader generality and flexibility, being applicable, in particular, to situations where existing tools are not directly useful.
\end{abstract}

\section{Introduction}

Since the early days of quantum mechanics, the concept of entanglement has proved a continuous source of physical, mathematical, and philosophical challenges. Interest in entanglement has heightened dramatically in recent years, the characterization and quantification of entanglement being nowadays one of the most active research areas within the emerging science of Quantum Information (QI) [NC]. So, what is the point about entanglement? Quoting from a landmark 1935 paper by Schrödinger, where the term entanglement was first introduced [] (see also [EPR]), "...This is the point. Whenever one has a complete expectation catalog - a maximum total knowledge - a $\psi$ function - for two completely separated bodies,... then one obviously has it also for the two bodies together... But the converse is not true. Maximal knowledge of a total system does not necessarily include total knowledge of all its parts, not even when these are fully separated from each other and at the moment are not influencing each other at all."

This can be interpreted as pointing out the possibility that some pure states of composite quantum systems - precisely those Schrödinger calls "entangled" - have mixed reduced states on their constituent subsystems. It is exactly this aspect of entanglement that we propose to generalize beyond the subsystem framework. Of course, a lot more has been learned by now about the properties and significance

1991 Mathematics Subject Classification. Primary 81P99; Secondary 81P68, 94A99.

Key words and phrases. Quantum mechanics, quantum information, entanglement.

Research sponsored by the US DOE. L.V. gratefully acknowledges support from the Los Alamos Office of the Director through a J.R. Oppenheimer Fellowship. This paper is based on a talk delivered at the Coding Theory and Quantum Computing Conference, University of Virginia, Charlottesville, May 20-24, 2003. 
of such states from both a physical and an information-theoretic perspective. In particular, because no pure state of a classical system can be correlated, the correlations present in a pure entangled state are intrinsically non-classical, as witnessed by the fact that the presence of entanglement is a necessary condition for the violation of Bell inequalities [BE, $\mathbf{P S}$. In addition, entangled states are known to provide the defining resource for quantum communication, enabling non-classical protocols such quantum teleportation and leading to provable improvements in the efficiency of various multipartite tasks [BU]. Finally, although a full assessment of the role of entanglement in quantum computational speed-up remains elusive to date, entangled states involving unboundedly many qubits turn out to be necessary for efficient pure-state quantum algorithms $\mathbf{J L}$.

In spite of such progress, there have been a number of signs recently that the theory of entanglement developed so far is too restrictive to be applicable to the full variety of both QI and physical settings one might like to consider. In particular, the conventional approach to entanglement focuses on analyzing how a quantum system is made up from constituent subsystems, and implicitly incorporates the assumption that the latter are operationally distinguishable. Compelling motivations for critically reconsidering the resulting entanglement notion come from situations where the operational access to the system of interest is inherently restricted by physical constraints. A first example is offered by condensed-matter systems, where the consequences of non-trivial (bosonic or fermionic) quantum statistics must be taken into account, making the direct identification of particles as "entanglable" subsystems problematic. A second example may occur in systems which are subject to superselection rules, which restrict the allowed transformations on the system's state space and effectively cause sectors of the latter to be operationally unreachable. Both situations are being actively investigated in the QI literature, and no attempt will be made here to provide a complete account. While different solutions have been examined thus far, they essentially all rely on either appropriately redefining the relevant subsystems to be used as primitive blocks, or the applicable measure for quantifying the accessible correlations - leaving, however, the underlying entanglement concept unchanged. We refer the reader to $\mathbf{O N}, \mathbf{E K}, \mathbf{Z F}$ and $\mathbf{B W}, \mathbf{V C}$ for representative discussions of entanglement for indistinguishable particles and in the presence of superselection rules, respectively.

Our approach takes a different route, bypassing the need of a subsystem decomposition in the first place, and reformulating entanglement as a property of quantum states which directly depends on physical observables. The investigation of the resulting notion of generalized entanglement (GE) was undertaken in [BKOV] and continued in BKOSV]. In the following, we further highlight some properties and implications of such a perspective.

\section{Entanglement and preferred subsystems}

Let $S$ be a quantum system with associate state space $\mathcal{H}, \operatorname{dim}(\mathcal{H})=d$, for some $d \in \mathbb{N}$. For simplicity, we shall focus on considering pure states of $S$ throughout. A subsystem of $S$ can be defined in general as a factor (in the tensor product sense) of a subspace $\mathcal{C} \subseteq \mathcal{H}[\mathbf{K L V}]$. In the simplest case where $\mathcal{C}=\mathcal{H}$, a subsystem decomposition of $S$ is described by a tensor product structure on $\mathcal{H}$,

$$
\mathcal{H} \simeq \bigotimes_{\ell} \mathcal{H}_{\ell}
$$


where $\mathcal{H}_{\ell}$ is the state space of the $\ell$ th subsystem. Whenever a multipartition of the form (2.1) is defined over a proper subspace of $\mathcal{H}$, an encoded tensor product structure is obtained, and the underlying subsystems are sometimes referred to as "logical" or "virtual" subsystems [ $\mathbf{Z}$.

Even in the simplest setting described by Eq. (2.1), it is essential to realize that, a priori, the number of inequivalent subsystem partitions that $\mathcal{H}$ can support may be large, reflecting the fact that many possibilities of expressing $d$ as a product of integers arise in general ${ }^{1}$. Accordingly, entanglement of a state in $\mathcal{H}$ is unambiguously defined only once a preferred subsystem decomposition is selected - which physically corresponds to a specific way of viewing $S$ as made up from constituent parts. Relative to a distinguished multipartite structure, a pure state $|\psi\rangle \in \mathcal{H}$ is entangled iff is not expressible as a product of states in $\mathcal{H}_{\ell}$. Given $|\psi\rangle$, the state of the $\ell$ th subsystem is determined by the reduced density operator $\rho_{\ell}$ resulting from the partial trace operation over the remaining subsystems. Thus, an equivalent characterization of standard pure-state entanglement is that $|\psi\rangle$ is entangled iff $\rho_{\ell}$ is mixed for some $\ell$ : entangled pure states are exactly those which appear mixed to observers whose operational access to $S$ is restricted to local observables acting on only a subsystem at a time.

The above observations suggest that the standard definition of entanglement can be equivalently phrased in terms of the observables used to measure the system and describe its states, and that perhaps this could serve as a starting point for an operational characterization of entanglement under more general circumstances. A natural path for capturing such an intuition is to relate the emergence of a preferred subsystem decomposition to be used to the set of available interactions and measurement capabilities. Such a path, which naturally extends the observalebased definition of subsystems in terms of associative algebras introduced in [KLV], VKL, Z, has been recently investigated by Zanardi et al. [ZLL]. The essence of our GE approach, as we shall see, is instead to redefine entanglement so as to make it directly dependent upon a physically relevant set of observables - irrespective of and without reference to a preferred way for partitioning $S$ into subsystems.

\section{The notion of generalized entanglement}

The key realization underlying the GE approach is that the distinctive features of entanglement are determined by the expectation values of a distinguished subspace of observables of $S$. The latter may directly incorporate an operational restriction, such as limited means for controlling and measuring the system, or more generally reflect an insight or condition dictated by Physics. Let such a preferred observable subspace be denoted by $\Omega$. The steps leading to GE may be summarized as follows:

- Pure states are unentangled (in the conventional sense) iff every subsystem state is pure;

- States can be represented as positive linear functionals on operator spaces, induced by a density operator according to the trace map. Formally, given a state of $S$ described by $\rho$, this completely specifies a positive linear

\footnotetext{
${ }^{1}$ See $\mathbf{Z}$ for a formal definition of equivalent factorizations and the resulting notion of tensorproduct-structure manifold.
} 
functional $\lambda$ on the space $\operatorname{End}(\mathcal{H})$ of all operators on $\mathcal{H}$ via the action

$$
\lambda(X)=\operatorname{tr}(\rho X), \quad X \in \operatorname{End}(\mathcal{H}),
$$

such that $\lambda\left(X^{\dagger} X\right) \geq 0$ and $\lambda(\mathbb{I})=1$. Such a state $\lambda$ can be restricted to $\Omega \subseteq \operatorname{End}(\mathcal{H})$, giving a reduced state $\omega=\lambda \mid \Omega$ which only determines the expectations of operators in $\Omega$. If, additionally, $\Omega$ is closed under Hermitian conjugation, we can define a reduced density operator $\rho_{\text {red }} \in \Omega$ associated with the state by the relationship

$$
\omega(Y)=\operatorname{tr}(\rho Y)=\operatorname{tr}\left(\rho_{\text {red }} Y\right), \quad Y \in \Omega
$$

- The set $\mathcal{R}$ of $\Omega$-reduced states is convex, that is, closed under probabilistic combinations;

- A state in $\mathcal{R}$ is pure iff it is extremal, that is, it cannot be expressed as a non-trivial convex combination of elements in $\mathcal{R}$.

We are thus led to the following:

Definition 3.1. A pure state $\rho=|\psi\rangle\langle\psi|$ of $S$ is generalized unentangled relative to the distinguished set of observables $\Omega$ if its reduced state is pure, and generalized entangled otherwise ${ }^{2}$.

While the above definition is applicable to an arbitrary linearly closed operator set $\Omega$, a relevant situation which is often encountered in physical applications occurs when $\Omega$ is a Lie algebra of operators acting on $\mathcal{H}$.

3.1. Lie-algebraic framework and Lie-algebraic purity. Let us focus on the case where $\Omega=\mathfrak{h}$ is a semisimple Lie algebra of operators acting irreducibly on the representation space $\mathcal{H}$. In order to make the connection with physical observables more transparent, we also assume that $\mathfrak{h}$ is a real Lie algebra consisting of Hermitian operators, with a modified Lie-bracket operation defined as follows:

$$
[X, Y]=i(X Y-Y X), \quad X, Y \in \mathfrak{h} .
$$

Accordingly, the Lie group $G$ generated by $\mathfrak{h}$ will be obtained via the modified exponential map $X \mapsto e^{i X}$. The reader is referred to [G, HA, HU] for relevant background on Lie representation theory. The general case of a reducible action of $\mathfrak{h}$ on $\mathcal{H}$ is addressed elsewhere (see for instance [SOBKV]).

Under the above assumptions, one of the key results established in BKOV] (see Theorem 14, part (3)) is the identification of the generalized unentangled pure states as the generalized coherent states (GCSs) associated with the Lie algebra $\mathfrak{h}$ (see also $\underline{\mathbf{K}}$ for a related characterization motivated by geometric invariant theory). A review of the theory underlying GCSs and their main physical applications may be found in [PR, ZFG]. Recall that GCSs are obtained by extending the definition of canonical (harmonic-oscillator) coherent states in terms of a displaced reference state within a general Lie-algebraic framework. Beside the Lie algebra $\mathfrak{h}$ with associated dynamical group $G$ acting on $\mathcal{H}$, the definition of GCSs requires the specification of a normalized reference state $\left|\Psi_{0}\right\rangle \in \mathcal{H}$ which, following Gilmore's construction, is chosen as an extremal (lowest-weight) state of the representation. Knowledge of $G$ and $\left|\Psi_{0}\right\rangle$ determines the subgroup $G_{0} \subseteq G$ of elements that preserve the reference state up to a phase factor. The manifold of GCSs associated to

\footnotetext{
${ }^{2}$ Similarly, a mixed state $\rho$ of $S$ is generalized unentangled relative to $\Omega$ if it can be written as a proper convex combination of generalized unentangled pure states.
} 
$\left(\mathfrak{h}, \mathcal{H},\left|\Psi_{0}\right\rangle\right)$ is then defined by the orbit of the reference state under coset elements in $G / G_{0}$ that is,

$$
\operatorname{GCS}_{\left(\mathfrak{h}, \mathcal{H},\left|\Psi_{0}\right\rangle\right)}=\left\{\mathcal{D}\left|\Psi_{0}\right\rangle \mid \mathcal{D} \in G / G_{0}\right\} .
$$

Accordingly, all generalized unentangled states are GCSs and are in the orbit of a (unique) minimum weight state of $\mathfrak{h}$ under the action of the Lie group.

Given a pure state $|\psi\rangle \in \mathcal{H}$, one would like to obtain a quantitative way for relating the properties of generalized unentanglement, generalized coherence, and purity with respect to the observable subspace. A natural procedure is to consider the projection of $|\psi\rangle\langle\psi|$ onto $\mathfrak{h}$, as the latter completely determines the expectation values of operators in the Lie algebra. This motivates the following:

Definition 3.2. Let $\left\{x_{i}\right\}$ be a Hermitian and commonly-normalized orthogonal $\left(\operatorname{tr} x_{i} x_{j} \propto \delta_{i j}\right)$ basis for $\mathfrak{h}$. For any $|\psi\rangle \in \mathcal{H}$, the purity of $|\psi\rangle$ relative to $\mathfrak{h}$ (or $\mathfrak{h}$-purity) is

$$
P_{\mathfrak{h}}(|\psi\rangle)=\sum_{i}\left(\operatorname{tr}|\psi\rangle\langle\psi| x_{i}\right)^{2}=\sum_{i}\left|\left\langle\psi\left|x_{i}\right| \psi\right\rangle\right|^{2} .
$$

Remark 3.3. Let $\mathcal{P}_{\mathfrak{h}}$ denote the projection map onto $\mathfrak{h}$. As defined, the $\mathfrak{h}$ purity of a state is the square length of the projection $\mathcal{P}_{\mathfrak{h}}(|\psi\rangle\langle\psi|)$ according to the trace-inner-product norm. Note that, in principle, different operator norms could be used, resulting in different purity functionals for fixed $\mathfrak{h}$.

REMARK 3.4. Let $D \in G$ be an arbitrary group transformation. Then $P_{\mathfrak{h}}(|\psi\rangle)=$ $\tilde{P}_{\mathfrak{h}}(|\psi\rangle)$, where the latter is calculated by replacing the operators $x_{i}$ with $D^{\dagger} x_{i} D$ in Eq. (3.2). Because $\tilde{P}_{\mathfrak{h}}(|\psi\rangle)=P_{\mathfrak{h}}(D|\psi\rangle)$, the $\mathfrak{h}$-purity is invariant under group transformations, as is desirable on physical grounds.

With the above definitions, the main features of GE within the Lie-algebraic framework are summarized by the following:

THEOREM 3.5. The following characterizations are equivalent for an irreducibly represented Lie algebra $\mathfrak{h}$ on $\mathcal{H}$ :

(1) $\rho$ is generalized unentangled relative to $\mathfrak{h}$.

(2) $\rho=|\psi\rangle\langle\psi|$ with $|\psi\rangle$ the unique ground state of some Hamiltonian $H$ in $\mathfrak{h}$.

(3) $\rho=|\psi\rangle\langle\psi|$ with $|\psi\rangle$ a lowest-weight vector of $\mathfrak{h}$.

(4) $\rho$ has maximum $\mathfrak{h}$-purity.

A proof of this Theorem is available in $\mathbf{B K O V}$. We also refer the reader to the same paper for an extended discussion of generalizations of various informationtheoretic notions (such as local maps and complexity measures) to the Lie-algebraic setting and beyond. In the following, we focus on illustrating some aspects of the relationship between conventional and generalized entanglement.

\section{Conventional entanglement revisited}

Given the quantum system $S$ and a pure state $|\psi\rangle \in \mathcal{H}$, the purity relative to the (real) Lie algebra of all traceless observables $\mathfrak{h}=\mathfrak{s u}(d)$ spanned by an orthogonal, commonly normalized Hermitian basis $\left\{x_{1} \cdots x_{L}\right\}, L=d^{2}-1$, is, according to Eq. (3.2), given by:

$$
P_{\mathfrak{h}}(|\psi\rangle)=\mathrm{K} \sum_{\alpha=1}^{L}\left\langle x_{\alpha}\right\rangle^{2},
$$


where the overall normalization $\mathrm{K}$ depends on the dimension $d$ and is determined by requiring that the maximum purity value is 1 . For an orthonormal operator basis, $\operatorname{Tr}\left(x_{\alpha} x_{\beta}\right)=\delta_{\alpha, \beta}, \mathrm{K}=d /(d-1)$, whereas in the case $\operatorname{Tr}\left(x_{\alpha} x_{\beta}\right)=d \delta_{\alpha, \beta}$ (as for ordinary un-normalized Pauli matrices $), K=1 /(d-1)$. Because any quantum state $|\psi\rangle \in \mathcal{H}$ can be obtained by applying a group operator $\mathcal{D}$ to a reference state $\mid$ ref $\rangle$ (a lowest-weight state of $\mathfrak{s u}(d)$ ),

$$
|\psi\rangle=\mathcal{D}|\mathrm{ref}\rangle, \quad \mathcal{D}=e^{i \sum_{\alpha} t_{\alpha} x_{\alpha}}, t_{\alpha} \in \mathbb{C},
$$

any quantum state $|\psi\rangle$ is a GCS of $\mathfrak{s u}(d)$, thus generalized unentangled relative to the full observable algebra: $P_{\mathfrak{h}}(|\psi\rangle)=1$ for all $|\psi\rangle$.

Let now assume that $S$ is composed of $n$ distinguishable susbsytems, corresponding to a state-space factorization of the form (2.1), that is, $\mathcal{H} \simeq \otimes_{\ell} \mathcal{H}_{\ell}$, with $\operatorname{dim}\left(\mathcal{H}_{\ell}\right)=d_{\ell}, \prod_{\ell} d_{\ell}=d$. In the conventional setting, distinguishability of the subsystems motivates the assumption of local accessibility to their individual state spaces. Thus, the set of local observables, consisting of operators which act on each subsystem independently, is physically distinguished. Let

$$
\mathfrak{h}_{\text {loc }}=\bigoplus_{\ell=1}^{n} \mathfrak{s u}\left(d_{\ell}\right)
$$

denote the corresponding (real) Lie algebra of traceless local observables, acting irreducibly on $\mathcal{H}$. Because standard unentangled pure states are exactly those for which all reduced states remain extremal, the GE notion relative to the local observable subspace coincides with the standard notion of pure state multipartite entanglement [BKOV]. This may be explicitly appreciated by studying the local purity $P_{\mathfrak{h}}$ and its relation to the conventional subsystem purities determined by the reduced subsystem states.

4.1. Local purities vs subsystem purities. An orthonormal basis which is suitable for calculating $P_{\mathfrak{h}}=P_{\mathfrak{h}_{\text {loc }}}$ may be obtained by considering a collection of orthonormal bases $\left\{x_{\alpha_{1}}^{\ell} \cdots x_{\alpha_{L_{\ell}}}^{\ell}\right\}, L_{\ell}=d_{\ell}^{2}-1$, each acting on the $\ell$ th factor alone, that is,

$$
x_{\alpha_{\ell}}^{\ell}=\overbrace{\mathbb{I}^{1} \otimes \mathbb{I}^{2} \otimes \cdots \otimes \underbrace{x_{\alpha_{\ell}}}_{\ell^{t h} \text { factor }} \otimes \cdots \otimes \mathbb{I}^{n}}^{n \text { factors }}
$$

where $\mathbb{I}^{\ell}=\mathbb{I} / \sqrt{d_{\ell}}$. Let also $\mathfrak{h}_{\ell}=\operatorname{span}\left\{x_{\alpha_{\ell}}\right\}$ be the Lie algebra of traceless Hermitian operators acting on $\mathcal{H}_{\ell}$ alone, and $\rho_{\ell}=\operatorname{Tr}_{\ell^{\prime} \neq \ell}(\{|\psi\rangle\langle\psi|\})$ the reduced density operator describing the $\ell$ th subsystem's state. We can then prove:

TheOREM 4.1. For every pure state $|\psi\rangle \in \mathcal{H}$ the following identities hold:

(1) For each $\ell$, the purity relative to the algebra $\mathfrak{h}_{\ell}$ is proportional to the conventional subsystem purity:

$$
P_{\mathfrak{h} \ell}(|\psi\rangle)=\frac{d_{\ell}}{d_{\ell}-1}\left(\operatorname{tr} \rho_{\ell}^{2}-\frac{1}{d_{\ell}}\right) .
$$


(2) The purity relative to the local algebra $\mathfrak{h}$ is proportional to the average subsystem purity:

$$
P_{\mathfrak{h}_{l o c}}(|\psi\rangle)=\frac{1}{1-\frac{1}{n} \sum_{\ell=1}^{n} \frac{1}{d_{\ell}}}\left[\frac{1}{n} \sum_{\ell=1}^{n}\left(\operatorname{tr} \rho_{\ell}^{2}-\frac{1}{d_{\ell}}\right)\right] .
$$

Proof. The state of the $\ell$ th subsystem may be represented as

$$
\rho_{\ell}=\frac{\mathbb{I}}{d_{\ell}}+\sum_{\alpha_{\ell}}\left\langle x_{\alpha_{\ell}}\right\rangle x_{\alpha_{\ell}},
$$

where $\left\langle x_{\alpha_{\ell}}\right\rangle=\operatorname{tr}\left(\rho_{\ell} x_{\alpha_{\ell}}\right)=\operatorname{tr}\left(|\psi\rangle\langle\psi| x_{\alpha_{\ell}}^{\ell}\right)$ and the last equality follows from the definition of reduced density operator. Thus,

$$
\operatorname{tr} \rho_{\ell}^{2}=\frac{1}{d_{\ell}}+\sum_{\alpha_{\ell}}\left\langle x_{\alpha_{\ell}}\right\rangle^{2} x_{\alpha_{\ell}} .
$$

By combining the above relation with the definition of the $P_{\mathfrak{h}_{\ell}}$-purity,

$$
P_{\mathfrak{h}_{\ell}}(|\psi\rangle)=\frac{d_{\ell}}{d_{\ell}-1} \sum_{\alpha_{\ell}=1}^{L_{\ell}}\left\langle x_{\alpha_{\ell}}\right\rangle^{2} x_{\alpha_{\ell}}
$$

Eq. 4.5 follows.

To derive the second equality, start from the definition of the $P_{\mathfrak{h}}$-purity which, using Eqs. (3.2) and (4.4), reads:

$$
P_{\mathfrak{h}}(|\psi\rangle)=\mathrm{K}^{\prime} \sum_{\ell=1}^{n} \sum_{\alpha_{\ell}=1}^{L_{\ell}}\left\langle x_{\alpha_{\ell}}^{\ell}\right\rangle^{2}=\mathrm{K}^{\prime} \sum_{\ell=1}^{n} \frac{d_{\ell}-1}{d_{\ell}} P_{\mathfrak{h}_{\ell}}(|\psi\rangle) .
$$

Clearly, the maximum value of the above quantity will be attained when, and only when, each of the terms $\operatorname{tr} \rho_{\ell}^{2}=1 \leftrightarrow P_{\mathfrak{h}_{\ell}}=1$ for all $\ell$, corresponding to a pure product state of the form $|\psi\rangle=\otimes_{\ell}\left|\psi_{\ell}\right\rangle$. This allows to determine the $\mathrm{K}^{\prime}-$ normalization factor as

$$
\mathrm{K}^{\prime}=\frac{1}{n-\sum_{\ell} \frac{1}{d_{\ell}}}=\frac{1}{n\left(1-\frac{1}{n} \sum_{\ell} \frac{1}{d_{\ell}}\right)},
$$

leading to the expression given in Eq. (4.6).

REMARK 4.2. Note that $1 / d_{\ell} \leq \operatorname{tr} \rho_{\ell}^{2} \leq 1$ for every $\ell$, corresponding to a totally mixed and pure reduced state, respectively. Thus, the quantity in round parentheses in Eq. (4.5) varies between 0 and $1-1 / d_{\ell}$, consistently implying a $P_{\mathfrak{h}_{\ell}}$-purity range between 0 and 1 .

REMARK 4.3. In the case of co-dimensional subsystems, $d_{\ell}=d_{0} \forall \ell$, Eq. (4.6) takes the simpler form

$$
P_{\mathfrak{h}}(|\psi\rangle)=\frac{d_{0}}{d_{0}-1}\left[\frac{1}{n} \sum_{\ell=1}^{n}\left(\operatorname{tr} \rho_{\ell}^{2}-\frac{1}{d_{0}}\right)\right]=\frac{1}{d_{0}-1}\left(\frac{d_{0}}{n} \sum_{\ell=1}^{n} \operatorname{tr} \rho_{\ell}^{2}-1\right) .
$$

According to the above Theorem, states corresponding to maximal local purity (i.e., generalized unentangled) are exactly the set of standard separable states. Note, however, that all pure states of $\mathcal{H}$ - whether they are conventionally unentangled or not relative to the selected state space decomposition - correspond to extremal reduced states relative to the full operator algebra $\mathfrak{g}=\mathfrak{s u}(d)$ : accordingly, 
all pure states of $\mathcal{H}$ are generalized unentangled relative to such algebra. Physically, this expresses the fact that no distinction between entangled and unentangled states is operationally meaningful if full access to arbitrary non-local operators is available. In a sense, the emergence of entanglement in this generalized perspective appears intimately and directly associated with physical constraints.

4.2. Multipartite qubit systems. The relevant case of $n$ qubits is recovered by letting $d_{\ell}=d_{0}=2 \forall \ell$, in which case one simply has $\mathrm{K}^{\prime}=2 / n$. A natural choice for calculating the local purity via Eq. (3.2) is obtained by choosing standard multi-qubit Pauli operators,

$$
\mathfrak{h}_{l o c}=\bigoplus_{\ell=1}^{n} \mathfrak{s u}(2)_{\ell}=\operatorname{span}\left\{\sigma_{x}^{1}, \sigma_{y}^{1}, \sigma_{z}^{1}, \cdots, \sigma_{x}^{n}, \sigma_{y}^{n}, \sigma_{z}^{n}\right\}
$$

where

$$
\sigma_{\alpha}^{\ell}=\overbrace{\mathbb{I} \otimes \mathbb{I} \otimes \cdots \otimes \underbrace{\sigma_{\alpha}}_{\ell^{t h} \text { factor }} \otimes \cdots \otimes \mathbb{I},}^{n \text { factors }}
$$

and the $2 \times 2$ matrices $\sigma_{\alpha}$ and $\mathbb{I}$ are the standard Pauli matrices, satisfying $\sigma_{\alpha}^{2}=\mathbb{I}$. By noticing that $x_{\alpha}=\sigma_{\alpha} / \sqrt{2}$ in this case, the purity of an arbitrary pure state becomes then

$$
P_{\mathfrak{h}}(|\psi\rangle)=\frac{1}{n} \sum_{\alpha=x, y, z} \sum_{\ell=1}^{n}\left\langle\sigma_{\alpha}^{\ell}\right\rangle^{2}
$$

As above, $P_{\mathfrak{h}}=1$ in any product state of the form $|\psi\rangle=\otimes_{\ell}\left|\psi_{\ell}\right\rangle$, a multi-qubit GCS in this algebra. Using Theorem 4.1, the relationships between local purities and qubit reduced states rewrite respectively as follows:

$$
\begin{gathered}
P_{\mathfrak{h}_{\ell}}(|\psi\rangle)=2\left(\operatorname{tr} \rho_{\ell}^{2}-\frac{1}{2}\right) \\
P_{\mathfrak{h}_{l o c}}(|\psi\rangle)=\frac{2}{n} \sum_{\ell=1}^{n}\left(\operatorname{tr} \rho_{\ell}^{2}-\frac{1}{2}\right)=\frac{2}{n} \sum_{\ell=1}^{n} \operatorname{tr} \rho_{\ell}^{2}-1 .
\end{gathered}
$$

EXAMPLE 4.4. With the above definitions, all pure product states of $n$ qubits have maximal purity that is, they are generalized unentangled. On the opposite limit, states of the form $\left|\mathrm{GHZ}_{n}\right\rangle=(|\uparrow \uparrow \ldots \uparrow\rangle+|\downarrow \downarrow \ldots \downarrow\rangle) / \sqrt{2}$ or of the form $[(|\uparrow \downarrow\rangle-|\downarrow \uparrow\rangle) / \sqrt{2}]^{\otimes n / 2}$ (for $n$ even) will be maximally entangled according to this measure $\left(P_{\mathfrak{h}}=0\right)$.

Because GE relative to the local algebra takes contributions, as quantified by the measure $P_{\mathfrak{h}}$, from correlations involving all subsystems simultaneously, one could expect such a measure to contain information about overall entanglement properties of the system (as opposed, for instance, to the concurrence measure which is intrinsically bipartite $[\mathbf{W}]$ ). A measure $Q$ of global entanglement for pure states of $n$ qubits was recently proposed by Meyer and Wallach in $\mathbf{M W}$, and shown to exhibit correct invariance properties under local unitary operations. According to the original construction, the first step to define such a measure is to consider a 
family of maps $l_{j}(b)$ given by the following action in the computational basis (and extended by linearity):

$$
l_{j}(b)\left|b_{1}, \cdots, b_{n}\right\rangle=\delta_{b b_{j}}\left|b_{1}, \cdots, \hat{b}_{j}, \cdots, b_{n}\right\rangle,
$$

where $b$ and $b_{j}$ are either $|0\rangle$ or $|1\rangle$, and $\hat{b}_{j}$ denotes the absence of the $j$ th qubit in the resulting $(n-1)$-qubit state. Let any pure state in $\left(\mathbb{C}^{2}\right)^{\otimes n-1}$ be represented in the computational basis, and let $|u\rangle=\sum u_{i}\left|\phi_{i}\right\rangle$ and $|v\rangle=\sum v_{i}\left|\phi_{i}\right\rangle$ be two such states. If a distance function on $\left(\mathbb{C}^{2}\right)^{\otimes n-1} \otimes\left(\mathbb{C}^{2}\right)^{\otimes n-1}$ is introduced as

$$
D(u, v)=\frac{1}{2} \sum_{i, j}\left|u_{i} v_{j}-u_{j} v_{i}\right|^{2}
$$

the global entanglement of a pure $n$-qubit state $|\psi\rangle$ is defined as follows:

$$
Q(|\psi\rangle)=\frac{4}{n} \sum_{j=1}^{n} D\left(l_{j}(0)|\psi\rangle, l_{j}(1)|\psi\rangle\right)
$$

Interestingly, as explained in BKOSV, SOBKV], the measures $P_{\mathfrak{h}}$ and $Q$ turn out to have a simple relationship.

THEOREM 4.5. Let $\mathfrak{h}$ denote the local observable algebra for $n$ qubits as defined above. Then for every pure state $|\psi\rangle \in \mathcal{H}=\left(\mathbb{C}^{2}\right)^{\otimes n}$ the following identity holds:

$$
P_{\mathfrak{h}}(|\psi\rangle)=1-Q(|\psi\rangle)
$$

Proof. By partitioning the $n$ qubits into the $j$ th qubit and the remaining one, and by working in the computational bases $\left\{\left|0_{j}\right\rangle,\left|1_{j}\right\rangle\right\}$ and $\left\{\left|\phi_{i}\right\rangle\right\}, i=1, \ldots, 2^{n-1}$ of the $j$ th qubit and the rest, respectively, any pure quantum state $|\psi\rangle \in\left(\mathbb{C}^{2}\right)^{\otimes n}$ can be represented as

$$
|\psi\rangle=\sum_{i=1}^{2^{n-1}}\left[g_{i}^{j}\left|0_{j}\right\rangle+h_{i}^{j}\left|1_{j}\right\rangle\right]\left|\phi_{i}\right\rangle, \quad j \in\{1, \ldots, n\},
$$

where $g_{i}^{j}$ and $h_{i}^{j}$ are complex coefficients. Therefore, the action of the map $l_{j}(b)$ on $|\psi\rangle$ given in Eq. (4.12) is

$$
\begin{aligned}
& l_{j}(0)|\psi\rangle=\sum_{i=1}^{2^{n-1}} g_{i}^{j}\left|\phi_{i}\right\rangle \\
& l_{j}(1)|\psi\rangle=\sum_{i=1}^{2^{n-1}} h_{i}^{j}\left|\phi_{i}\right\rangle .
\end{aligned}
$$

Using Eq. (4.13), the distance between the above states of $(n-1)$ qubits becomes

$$
D\left(l_{j}(0)|\psi\rangle, l_{j}(1)|\psi\rangle\right)=\sum_{i, i^{\prime}}\left[\left|g_{i}^{j}\right|^{2}\left|h_{i^{\prime}}^{j}\right|^{2}-\left(g_{i}^{j} h_{i^{\prime}}^{j}\right)\left(h_{i}^{j} g_{i^{\prime}}^{j}\right)^{*}\right],
$$


where ${ }^{*}$ denotes complex conjugate. After some algebraic manipulations we get

$$
\begin{aligned}
& \sum_{i=1}^{2^{n-1}}\left|g_{i}^{j}\right|^{2}=\left\langle\psi\left|\left(\frac{1+\sigma_{z}^{j}}{2}\right)\right| \psi\right\rangle, \\
& \sum_{i=1}^{2^{n-1}}\left|h_{i}^{j}\right|^{2}=\left\langle\psi\left|\left(\frac{1-\sigma_{z}^{j}}{2}\right)\right| \psi\right\rangle, \\
& \sum_{i=1}^{2^{n-1}} g_{i}^{j}\left(h_{i}^{j}\right)^{*}=\left\langle\psi\left|\sigma_{-}^{j}\right| \psi\right\rangle,
\end{aligned}
$$

thus Eq. (4.17) rewrites as

$$
D\left(l_{j}(0)|\psi\rangle, l_{j}(1)|\psi\rangle\right)=\frac{1}{4}\left[1-\left\langle\sigma_{z}^{j}\right\rangle^{2}-\left\langle\sigma_{x}^{j}\right\rangle^{2}-\left\langle\sigma_{y}^{j}\right\rangle^{2}\right] .
$$

By taking a sum over all qubits and recalling Eq. (4.14), we finally obtain the desired result

$$
Q(|\psi\rangle)=1-\frac{1}{n} \sum_{j=1}^{n}\left[\left\langle\sigma_{z}^{j}\right\rangle^{2}+\left\langle\sigma_{x}^{j}\right\rangle^{2}+\left\langle\sigma_{y}^{j}\right\rangle^{2}\right]=1-P_{\mathfrak{h}}(|\psi\rangle) .
$$

Theorem 4.5 together with Eq. (4.11) also implies a direct, linear relationship between the global entanglement metric $Q$ of $[\mathbf{M W}]$ and the average subsystem purity, that is,

$$
Q(|\psi\rangle)=2\left(1-\frac{1}{n} \sum_{\ell=1}^{n} \operatorname{tr} \rho_{\ell}^{2}\right) .
$$

The above relationship has been independently established by Brennen [BR].

\section{Generalized entanglement and subsystems}

According to the results of the previous section, GE includes conventional entanglement as a special case: when a given partition of $\mathcal{H}$ into subsystems is specified, there exists a natural observable subspace (the one describing local actions on individual factors) such that entanglement relative to the given subsystem partition and GE relative to the associated local algebra coincides for all states. Suppose, however, that the opposite point of view is taken, namely that the state space $\mathcal{H}$ of a quantum system is known, along with a distinguished, irreducible Lie algebra $\mathfrak{h}$ (or, more generally, an observable set $\Omega$ ). Then the following questions arise: Are there preferred subsystem partitions on $\mathcal{H}$ which may be determined by $\mathfrak{h}$ ? If so, does a (possibly fictitious) subsystem partition exist, such that generalized entangled states relative to $\mathfrak{h}$ are exactly the set (or are a subset) of ordinary entangled states relative to such a partition? In other words, does GE represent a genuine extension of the subsystem-based entanglement framework in its most general formulation?

From the physical point of view, the novelty and added generality afforded by the GE notion should be already clear from the direct applicability of our prescription to any situation where a laboratory condition or physical constraint singles out a special observable set, shortcutting the need for any intermediate subsystem identification. From a mathematical perspective, a distinctive innovation with respect to the traditional framework is that GE ultimately depends only on the convexity 
properties of observable sets [BKOV]. The the resulting extremality characterization of unentangled states has not been emphasized in the conventional setting (for instance, it is not part of [ZLL]'s treatment of the virtual-subsystem approach). With these general considerations in mind, however, a thorough analysis of the relationships between our approach and the subsystem-based one is an interesting issue worth investigating per se. We limit ourselves here to a few illustrative remarks.

5.1. Observable-dependent subsystems. If, as assumed so far, the distinguishable observables form a semisimple Lie algebra $\mathfrak{h}$, a natural multipartite setting is determined by the fact that $\mathfrak{h}$ is uniquely expressible as a product of simple Lie algebras $\mathfrak{h}=\times_{k} \mathfrak{h}_{k}[\mathbf{H U}]$. A Hilbert space irreducibly representing $\mathfrak{h}$ then factors as ${ }^{3} \mathcal{H}=\otimes_{k} \mathcal{H}_{k}$, with the algebra $\mathfrak{h}_{k}$ acting as the identity over all factors except the $k$ th one. In the language of $\mathbf{Z L L}$, the resulting multipartition is formally reminiscent of an observable-induced tensor product structure associated to $\left\{\mathfrak{h}_{k}\right\}$ over $\mathcal{H}$. Similarly, a formal analogy with the emergence of encoded multipartitions $(\mathcal{C} \subset \mathcal{H})$ may be expected for a reducible representation of $\mathfrak{h}$ on $\mathcal{H}$. Note, however, that actions on the individual state spaces $\mathcal{H}_{k}$ belong to a Lie group representation which need not be $\mathrm{GL}\left(\operatorname{dim}\left(\mathcal{H}_{k}\right)\right)$ as for standard entanglement theory BKOV].

5.2. Entanglement without subsystems. A situation which strikingly illustrates the added flexibility of the GE notion is offered by quantum systems whose states space is intrinsically irreducible, that is, $d$ is a prime number. In this case, the system is physically elementary, and conventional entanglement is not directly applicable. Consider for instance a single spin-1 system, whose three-dimensional state space $\mathcal{H}=\mathbb{C}^{3}$ carries an irreducible representation of $s u(2)$, with usual angular momentum generators $J_{x}, J_{y}, J_{z}$ given by

$$
J_{x}=\frac{1}{\sqrt{2}}\left(\begin{array}{lll}
0 & 1 & 0 \\
1 & 0 & 1 \\
0 & 1 & 0
\end{array}\right), J_{y}=\frac{1}{\sqrt{2}}\left(\begin{array}{ccc}
0 & -i & 0 \\
i & 0 & -i \\
0 & i & 0
\end{array}\right), J_{z}=\frac{1}{\sqrt{2}}\left(\begin{array}{ccc}
1 & 0 & 0 \\
0 & 0 & 0 \\
0 & 0 & -1
\end{array}\right)
$$

Suppose that, due to operational limitations, observations on this system are restricted to observables linear in the above generators of $\mathfrak{s u}(2)$. Given an arbitrary pure state $|\psi\rangle \in \mathbb{C}^{3}$, the corresponding reduced state can be identified with the vector of expectation values of these three observables $\left\langle J_{\alpha}\right\rangle$. The set of such reduced states is a unit ball in $\mathbb{R}^{3}$, defined by $\left\langle J_{x}\right\rangle^{2}+\left\langle J_{y}\right\rangle^{2}+\left\langle J_{z}\right\rangle^{2} \leq 1$. The extremal states correspond to points on the surface, resulting from maximal spin component 1 for some linear combination of $J_{x}, J_{y}, J_{z}$. For any choice of spin direction, $\mathcal{H}$ is spanned by the $|1,+1\rangle,|1,0\rangle,|1,-1\rangle$ eigenstates of that spin component, for instance the $\hat{z}$ direction. Then the extremal states are immediately identified with the spin coherent states $|1, \xi\rangle$, or GCSs for $\mathrm{SU}(2)$ [ZFG],

$$
|1, \xi\rangle=\mathrm{e}^{\xi J_{+}-\xi^{*} J_{-}}|1,-1\rangle, \quad \xi \in \mathbb{C},
$$

where the exponential involving the ladder operators $J_{ \pm}$provides an explicit realization of the group-displacement $\mathcal{D}$ of Eq. (3.1), and the lowest-weight state $\left|J=1, J_{z}=-1\right\rangle$ is chosen as the reference state. Note that the states $|1,+1\rangle$ and $|1,-1\rangle$ are GCSs, but $|1,0\rangle$ is not: thus, the latter is a generalized (in fact, maximally) entangled state relative to $\mathfrak{s u}(2)$. As also remarked earlier, access to the full operator algebra $(\mathfrak{s u}(3)$ in this case) causes GE to disappear altogether,

\footnotetext{
${ }^{3}$ Note that this implies $d=\operatorname{dim}(\mathcal{H})$ to be a non-prime integer.
} 
expressing the fact that arbitrary state vectors can be connected through group transformations in $\mathrm{SU}(3)$.

Note that, in principle, one could formally regard the single spin-1 system considered above as arising from two spin- $1 / 2$ subsystems in a triplet configuration. Then states which are generalized unentangled (or entangled) relative to $\mathfrak{s u}(2)$ would be associated with ordinary product (or entangled) states of the two fictitious subsystems,

$$
\begin{aligned}
|1,-1\rangle & \leftrightarrow|\downarrow\rangle_{1} \otimes|\downarrow\rangle_{2} \\
|1,0\rangle & \leftrightarrow \frac{|\uparrow\rangle_{1} \otimes|\downarrow\rangle_{2}+|\downarrow\rangle_{1} \otimes|\uparrow\rangle_{2}}{\sqrt{2}} \\
|1,+1\rangle & \leftrightarrow|\uparrow\rangle_{1} \otimes|\uparrow\rangle_{2},
\end{aligned}
$$

with self-explaining notation. We would like to stress that, from a physical point of view, such a partition is entirely artificial if operational access to the individual subsystems is unavailable as assumed above. Moreover, the procedure is everything but straightforward mathematically. Formally, the above line of reasoning requires embedding the original spin- 1 irreducible representation of $\mathfrak{s u}(2)$ in the tensor product representation of two spin- $1 / 2$ irreducible representations of $\mathfrak{s u}(2)$, in such a way that generalized entangled states becomes a subset of the ordinary entangled states in the extended representation ${ }^{4}$. While the simplicity of the state identification given above is coincidental to the elementary irreducible-representation-structure of this example, understanding to what extent a similar procedure could be carried out for general irreducible representations of semisimple Lie algebras might shed further light on the mathematical relationships between GE and abstract subsystem structures (See also discussion in Section 5).

\section{Discussion}

6.1. Implications for condensed-matter systems. A feature which makes the GE framework particularly attractive for applications to condensed-matter systems is the possibility to directly formulate entanglement in terms of the algebraic operator language (fermionic, bosonic, or other $[\mathbf{B O}$ ) which best describes the system. Among such applications, the possibility of gaining a better understanding of the nature and properties of the quantum correlations in a system undergoing a quantum phase transition has attracted a growing interest recently, see for instance ON, OAFF, VLRK. The usefulness of the GE framework in the context of identifying and characterizing quantum phase transitions has been investigated based on the explicit analysis of two integrable models undergoing a broken symmetry quantum phase transition - the well known one-dimensional spin-1/2 XY model in a transverse magnetic field and the so-called Lipkin-Meshkov-Glick model, respectively. We refer the interested reader to BKOSV, OSBKV, SOBKV] for detailed discussions. As these studies reveal, the purity relative to an appropriate Lie subalgebra of observables of the system provides a useful diagnostic tool for characterizing the many-body correlations which play a dominant role at criticality, by succeeding at both sharply detecting the critical point and correctly identifying the underlying universality class.

\footnotetext{
${ }^{4}$ Clearly, the (conventionally) entangled state spanning the singlet sector has no counterpart in the original physical space $\mathcal{H}$.
} 
While the above results are very suggestive and promising, several important questions remain to be investigated in more depth. In particular, a general operational criterion for identifying the physically relevant observable subalgebra for Hamiltonians whose ground-state properties are not easily computable is still lacking. The identification of a minimal collection of observable algebras able to provide a complete description of the system's critical properties is likewise an open problem. Finally, the extension of similar concepts and techniques to more general classes of quantum phase transitions (notably, topological quantum phase transitions) seems likely to require more sophisticated tools than the Lie-algebraic ones which suffice when a broken symmetry exists.

6.2. Outlook and conclusion. We have presented a generalization of entanglement which provides a subsystem-independent, unifying conceptual framework for defining entanglement in arbitrary physical settings. Unlike the conventional definition which is relative to a preferred decomposition into subsystem, GE is directly regarded as an observer-dependent property of quantum states, which is definable relative to any physically relevant set of observables for the system. Whenever the latter possess a Lie-algebraic structure, our approach naturally links entanglement theory with the theory of generalized coherent states.

In addition to the condensed-matter implications mentioned above, numerous information-theoretic problems also deserve further investigation. Several issues concerning appropriate generalizations of local maps, resource scaling, and GE measures have been raised and partially addressed in [BKOV]. Additional research directions might involve exploring possible connections between the presence of GE and the violation of Bell-type inequalities [PS], assessing the potential of GE detection via appropriate witness operators $[\mathbf{H O}, \mathbf{T}$, looking at possible characterizations of GE via uncertainty relations as recently suggested for conventional entanglement $[\mathbf{H T}$, and more. Ultimately, the fresh perspective offered by our approach will deepen our understanding of entanglement as a physical and information-theoretic resource.

\section{References}

[BE] J. S. Bell, Speakable and Unspeakable in Quantum Mechanics (Cambridge University Press, Cambridge, 1993).

[BO] C. D. Batista and G. Ortiz, Phys. Rev. Lett. 86, 1082 (2001); C. D. Batista, G. Ortiz, and J. E. Gubernatis, Phys. Rev. B 65, 180402 (2002).

[BR] G. K. Brennen, quant-ph/0305094 Quantum Inf. Comp. 3, 619 (2003).

[BDSW] C. H. Bennett, D. P. DiVincenzo, J. Smolin, and W. Wootters, Phys. Rev. A 54, 3824 (1996).

[BKOSV] H. Barnum, E. Knill, G. Ortiz, R. Somma, and L. Viola, quant-ph/0305023 Phys. Rev. Lett. 92 (2004), in press.

[BKOV] H. Barnum, E. Knill, G. Ortiz, and L. Viola, Phys. Rev. A 68, 032308 (2003).

[BU] H. Buhrman, R. Cleve, and W. Van Dam, SIAM J. Comp. 30, 1829 (2000).

[BW] S. D. Bartlett and H. M. Wiseman, Phys. Rev. Lett. 91, 097903 (2003).

[EK] K. Eckert, J. Schliemann, D. Bruss, and M. Lewenstein, Ann. Phys. 299, 88 (2002), and references therein.

[EPR] A. Einstein, B. Podolsky, and N. Rosen, Phys. Rev. 47, 777 (1935).

[G] H. Georgi, Lie Algebras in Particle Physics (Perseus Books, Reading, Massachusetts, 1999).

[HA] B. C. Hall, Lie Groups, Lie Algebras, and Representations (Springer-Verlag, New York, 2003).

[HO] H. Horodecki, P. Horodecki, and R. Horodecki, Phys. Lett. A 223, 1 (1996).

[HT] H. F. Hofmann and S. Takeuchi, Phys. Rev. A 68, 032103 (2003). 
[HU] J. E. Humphreys, Introduction to Lie Algebras and Representation Theory (Springer-Verlag, New York, 1972).

[JL] R. Jozsa and N. Linden, Proc. R. Soc. London A 459, 2011 (2003).

[K] A. Klyachko, quant-ph/0206012

[KLV] E. Knill, R. Laflamme, and L. Viola, Phys. Rev. Lett. 84, 2525 (2000).

[MW] D. A. Meyer and N. R. Wallach, J. Math. Phys. 43, 4273 (2002).

[NC] M. A. Nielsen and I. L. Chuang, Quantum Computation and Quantum Information (Cambridge University Press, Cambridge, 2000).

[OAFF] A. Osterloh, L. Amico, G. Falci, and R. Fazio, Nature 416, 608 (2002).

[ON] T. J. Osborne and M. A. Nielsen, Quant. Inf. Process. 1, 45 (2002); Phys. Rev. A 66, 032110 (2002).

[OSBKV] G. Ortiz, R. Somma, H. Barnum, E. Knill, L. Viola, "Entanglement as an observerdependent concept: An application to quantum phase transitions," quant-ph/0403043 to appear in Condensed Matter Theories, Vol. 19 (Nova Science Publishers, 2004).

[PR] A. Perelomov, Generalized Coherent States and their Applications (Springer-Verlag, Berlin, 1985).

[PS] A. Peres, Found. Phys. 29, 589 (1999).

[S] E. Schrödinger, Naturwissenschaften 23, 807 (1935). English translation available in Proc. Am. Phil. Soc 124, 323 (1980).

[SOBKV] R. Somma, G. Ortiz, H. Barnum, E. Knill, and L. Viola, "Nature and measure of entanglement in quantum phase transitions," quant-ph/0403035

[T] B. M. Terhal, Phys. Lett. A 271, 319 (2000).

[VC] F. Verstraete and J. I. Cirac, Phys. Rev. Lett. 91, 010404 (2003).

[VLRK] G. Vidal, J. I. Latorre, E. Rico, and A. Kitaev, Phys. Rev. Lett. 90, 227902 (2003).

[VKL] L. Viola, E. Knill, and R. Laflamme, J. Phys. A 34, 7067 (2001).

[W] W. Wootters, Phys. Rev. Lett. 80, 2245 (1998).

[Z] P. Zanardi, Phys. Rev. Lett. 87, 077901 (2001).

[ZF] P. Zanardi, Phys. Rev. A 65, 042101 (2002).

[ZFG] W. M. Zhang, D. H. Feng, and R. Gilmore, Rev. Mod. Phys. 62, 867 (1990).

[ZLL] P. Zanardi, D. A. Lidar, and S. Lloyd, quant-ph/0308043

Los Alamos National Laboratory, Los Alamos, New Mexico 87545

E-mail address: lviola@lanl.gov

Los Alamos National Laboratory, Los Alamos, New Mexico 87545

E-mail address: barnum@lanl.gov

National Institute of Standards and Technology, Boulder, Colorado 80305

E-mail address: knill@boulder.nist.gov

Los Alamos National Laboratory, Los Alamos, New Mexico 87545

E-mail address: ortiz@viking.lanl.gov

Los Alamos National Laboratory, Los Alamos, NM 87545, USA

E-mail address: somma@viking.lanl.gov 